\title{
ELEMENTARY PROOFS OF THE EXTREMAL PROPERTIES OF THE EIGENVALUES OF THE STURM-LIOUVILIE EQUATION
}

\author{
Paul R. Beesack \\ (received July 2,1959 )
} problem

1. Introduction. If the Sturm-Liouville eigenvalue

$$
\left(p y^{\prime}\right)^{\prime}+(q+\lambda g) y=0, y(a)=y(b)=0,
$$

is first approached from the standpoint of differential equations theory - as opposed, say, to the calculus of variations, or the theory of integral equations - the extremal properties of the eigenvalues seem to be generally regarded as lying beyond the scope of the theory. Thus, neither in the standard work of Bocher [1], nor in the recent work of Coddington and Levinson [2] is any mention made of this topic. Collatz [3, 166-8] gives an elementary proof of the minimum property of the least positive eigenvalue of (1.1), and a brief indication of how this argument can be extended to the higher eigenvalues. The purpose of this paper is to consolidate this elementary approach, and to extend it to cover the singular cases where either the interval is infinite, or one or more of the coefficients are singular at the end-points.

2. The principal integral inequality. The extremal properties of the eigenvalues of the Sturm-Liouville equation can all be based on the following theorem.

THEOREM 2.1. Let $p(x)$ and $G(x)$ be continuous, and $p(x)>0$ for $a<x<b$. Suppose the self-adjoint equation

$$
\left(p y^{\prime}\right)^{\prime}+G y=0
$$

has a solution $y(x) \neq 0$ on $a<x<b$, and that

Can. Math. Bull. vol. 3, no. 1, Jan. 1960 
(2.2) $(x-a) y^{\prime}(x) / y(x)=O(1)$ as $x \rightarrow a+,(b-x) y^{\prime}(x) / y(x)=O(1)$ as $x \rightarrow b-$.

If both of the conditions

$(2.3)_{1} \quad \mathrm{p}(\mathrm{x})=\mathrm{O}(\mathrm{x}-\mathrm{a})$ or $\mathrm{p}(\mathrm{x}) \int_{\mathrm{a}}^{\mathrm{x}} \mathrm{p}^{-1}(\mathrm{t}) \mathrm{dt}=\mathrm{O}(\mathrm{x}-\mathrm{a})$ as $\mathrm{x} \rightarrow \mathrm{at}$,

$(2.3)_{2} \quad p(x)=O(b-x)$ or $p(x) \int_{x}^{b} p^{-1}(t) d t=O(b-x)$ as $x \rightarrow b-$, hold, then

(2.4) $\lim _{a^{\prime} \rightarrow a,} b^{\prime} \rightarrow b \int_{a^{\prime}}^{b^{\prime}} G(x) u^{2}(x) d x \leqslant \int_{a}^{b} p(x) u^{\prime 2}(x) d x$

holds for any function $u(x)$ whose derivative is (absolutely) integrable on $a \leqslant x \leqslant b$, and for which

$$
u(a)=u(b)=0, \int_{a}^{b} p u^{\prime 2} d x<\infty .
$$

Before proving the theorem, we point out that it is valid if $a$ or $b$, or both, are infinite, provided the order conditions are modified to read

and

$$
x \cdot y^{\prime}(x) / y(x)=O(1) \text { as }|x| \rightarrow \infty,
$$

$$
p(x)=O(x) \text { or } p(x) \int_{-\infty}^{x} p^{-1}(t) d t=O(x) \text { as } x \rightarrow-\infty \text {, }
$$

with a similar condition at $b$, if $b$ is infinite. In the case that $a$ is finite, the first of conditions (2.2) will be assured if $y(x)$ is continuous on $a \leqslant x \leqslant b$, and if $\lim _{x \rightarrow a+} y^{\prime}(x)$ exists.

To prove the theorem, set $h=y^{1} / y$, and note that $h(x)$ satisfies the Riccati equation

$$
(\mathrm{ph})^{\prime}=-\mathrm{G}-\mathrm{ph} \mathrm{ph}^{2}, \mathrm{a}<\mathrm{x}<\mathrm{b} \text {. }
$$

Now for any $a^{\prime}, b^{\prime}$ with $a<a^{\prime}<b^{\prime}<b$, consider the integral

$$
\begin{aligned}
I\left(a^{\prime}, b^{\prime}\right) & =\int_{a^{\prime}}^{b^{\prime}} p\left(u^{\prime}-h u\right)^{2} d x \\
& =\int_{a^{\prime}}^{b^{\prime}} p u^{\prime 2} d x-2 \int_{a^{\prime}}^{b^{\prime}} \text { phuu'd } x+\int_{a^{\prime}}^{b^{\prime}} p h^{2} u^{2} d x,
\end{aligned}
$$

where $u(x)$ is any admissible function, i.e., any function satisfy- 
ing the hypotheses following (2.4). These integrals all exist since $p(x)$ and $h(x)$ are continuous on $a^{\prime} \leqslant x \leqslant b^{\prime}$. Also, since $\mathrm{p}(\mathrm{x})>0$ on $\mathrm{a}<\mathrm{x}<\mathrm{b}$, it follows that

(2.8) $\quad I\left(a^{\prime}, b^{\prime}\right)>0$ unless $u^{\prime}(x) \equiv h(x) u(x), \quad a^{\prime} \leqslant x \leqslant b^{\prime}$,

that is, unless $u(x) \equiv c y(x)$ on $a^{\prime} \leqslant x \leqslant b^{\prime}$. Moreover if $I\left(a^{\prime}, b^{\prime}\right)>0$ for any fixed $a^{\prime}, b^{\prime}$, then we clearly have

$$
\lim _{a^{\prime} \rightarrow a,} b^{\prime} \rightarrow b I\left(a^{\prime}, b^{\prime}\right)>0
$$

$($ possibly $+\infty)$. Integrating by parts the second term in the above expansion of $I\left(a^{\prime}, b^{\prime}\right)$, and making use of $(2.6),(2.7)$, we obtain

$$
I\left(a^{\prime}, b^{\prime}\right)=\int_{a^{\prime}}^{b^{\prime}} p u^{\prime 2} d x-\int_{a^{\prime}}^{b^{\prime}} G u^{2} d x-\left.p h u^{2}\right|_{a^{\prime}} ^{b^{\prime}}
$$

Hence

$$
\int_{a^{\prime}}^{b^{\prime}} G u^{2} d x \leqslant \int_{a^{\prime}}^{b^{\prime}} p u^{\prime 2} d x-\left.p h u^{2}\right|_{a^{\prime}} ^{b^{\prime}},
$$

where equality holds if, and only if, $u(x) \equiv c y(x)$.

We shall now prove that

(2.10) $\quad \lim _{a^{\prime} \rightarrow a} p^{2} u^{2}=0$, and $\lim _{b^{\prime} \rightarrow b} p u^{2}=0$.

We deal with the case $a^{\prime} \rightarrow a$, the other proof being identical. If the first of conditions (2.3) 1 holds, then using this and (2.2) we obtain

$$
\left|\mathrm{phu}^{2}\right| \leqslant \mathrm{k}_{1} \mathrm{k}_{2} \mathrm{u}^{2}
$$

for $x$ near at, so that (2.10) follows from (2.5). If the second of conditions $(2.3)_{1}$ holds, we use the fact that

$$
u(x)=\int_{a}^{x} u^{\prime}(t) d t \text {. }
$$

Hence,

$$
\begin{aligned}
|u(x)| & \leqslant \int_{a}^{x}\left|u^{\prime}\right| d t=\int_{a}^{x}\left(p^{\frac{1}{2}}\left|u^{\prime}\right|\right) \cdot\left(p^{-\frac{1}{2}}\right) d t \\
& \leqslant\left(\int_{a}^{x} p u^{\prime 2} d t\right)^{\frac{1}{2}}\left(\int_{a}^{x} p^{-1} d t\right)^{\frac{1}{2}}
\end{aligned}
$$


so

$$
u^{2}(x) \leqslant k_{3}(x) \int_{a}^{x} p^{-1} d t,
$$

where $k_{3}(x) \rightarrow 0$ as $x \rightarrow a+$ by the second of conditions (2.5). Thus, using $(2.3)_{1}$ we obtain

$$
\left|\mathrm{phu}^{2}\right| \leqslant \mathrm{k}_{1} \mathrm{k}_{4} \mathrm{k}_{3}(\mathrm{x})
$$

so (2.10) also follows in this case.

Using (2.10), the inequality (2.4) now follows from (2.9) on letting $a^{\prime} \rightarrow a$ and $b^{\prime} \rightarrow b$. By the remarks following (2.8), equality can hold in (2.4) only if $u(x) \equiv c y(x)$. However, cy(x) may not be admissible.

COROLLARY 2.1.1. Equality holds in (2.4) if, and only if, $u(x) \equiv c y(x)$, where $c=0$ unless $y^{\prime}(x)$ is absolutely integrable on $a \leqslant x \leqslant b$, and

$$
y(a)=y(b)=0, \quad \int_{a}^{b} p y^{12} d x<\infty .
$$

COROLLARY 2.1.2. If either $y(x)$ is admissible, or if $G(x) \geqslant 0$ (but $G \neq 0$ ), and $y(x)$ is continuous on $a \leqslant x \leqslant b$ with $y(a)=y(b)$, and either

$$
\int_{a}^{b^{\prime}} \mathrm{py}^{\prime 2} \mathrm{dx}=\infty \text {, or } \int_{\mathrm{a}^{\prime}}^{b} \mathrm{py}^{\prime 2} \mathrm{dx}=\infty,
$$

and

$$
\overline{\lim }_{x \rightarrow a} \mid \text { pyy }\left|<\infty, \overline{\lim }_{x \rightarrow b}\right| \text { Pyy } \mid<\infty,
$$

then the inequality (2.4) is best possible in the sense that the unit constant on the right side of (2.4) cannot be replaced by any smaller factor.

The corollary is obviously valid if $y(x)$ is admissible; hence we suppose $G(x) \geqslant 0, y(a)=y(b)$, and

$$
\int_{a}^{b^{\prime}} \text { py }^{12} \mathrm{dx}=\infty
$$

as well as the conditions (2.13). In this case, let $u(x)$ be defined by

$$
u(x)= \begin{cases}0, & a \leq x \leq a^{\prime} \\ y(x)-y\left(a^{\prime}\right), & a^{\prime} \leq x \leq b^{\prime} \\ 0, & b^{\prime} \leq x \leq b\end{cases}
$$


where $a^{\prime}$ and $b^{\prime}$ will be assigned later, and in such a way that $y\left(a^{\prime}\right)=y\left(b^{\prime}\right)$. This $u(x)$ is an admissible function, and using (2.9) and (2.1) we have

$$
\begin{aligned}
& \int_{a^{\prime}}^{b^{\prime}} G u^{2} d x=\int_{a^{\prime}}^{b^{\prime}} G\left[y(x)-y\left(a^{\prime}\right)\right]^{2} d x \\
= & \int_{a^{\prime}}^{b^{\prime}} G y^{2} d x-2 y\left(a^{\prime}\right) \cdot \int_{a^{\prime}}^{b^{\prime}} G y d x+y^{2}\left(a^{\prime}\right) \cdot \int_{a^{\prime}}^{b^{\prime}} G^{2} d x \\
= & \int_{a^{\prime}}^{b^{\prime}} p y^{\prime 2} d x-\left.p h y^{2}\right|_{a^{\prime}} ^{b^{\prime}}+2 y\left(a^{\prime}\right) \cdot \int_{a^{\prime}}^{b^{\prime}}\left(p y^{\prime}\right)^{\prime} d x+y^{2}\left(a^{\prime}\right) \cdot \int_{a^{\prime}}^{b^{\prime}} G^{2} d x .
\end{aligned}
$$

Hence,

$$
\begin{aligned}
& \int_{a}^{b} G u^{2} d x \geqslant \int_{a^{\prime}}^{b^{\prime}} p y^{\prime} 2 d x-\left.p y y^{\prime}\right|_{a^{\prime}} ^{b^{\prime}}+\left.2 y\left(a^{\prime}\right)\left(p y^{\prime}\right)\right|_{a^{\prime}} ^{b^{\prime}} \\
= & \int_{a}^{b} p u^{\prime} 2 d x-p\left(a^{\prime}\right) y\left(a^{\prime}\right) y^{\prime}\left(a^{\prime}\right)-p\left(b^{\prime}\right) y\left(b^{\prime}\right) y^{\prime}\left(b^{\prime}\right)+2 y\left(a^{\prime}\right) p\left(b^{\prime}\right) y^{\prime}\left(b^{\prime}\right) .
\end{aligned}
$$

Given $\delta(0<\delta<1)$, this last expression will exceea $(1-\delta) \int_{a}^{b} p^{\prime 2} d x$, provided

$$
\delta \int_{a^{\prime}}^{b^{\prime}} p y^{\prime 2} d x>p\left(a^{\prime}\right) y\left(a^{\prime}\right) y^{\prime}\left(a^{\prime}\right)+p\left(b^{\prime}\right) y\left(b^{\prime}\right) y^{\prime}\left(b^{\prime}\right)-2 y\left(a^{\prime}\right) p\left(b^{\prime}\right) y^{\prime}\left(b^{\prime}\right) .
$$

We will shortly show that, given a' sufficiently close to $a$, there exists $b^{\prime}<b$ such that $y\left(a^{\prime}\right)=y\left(b^{\prime}\right)$. Assuming this for now, our result will be proved if we can choose a' so that

$$
\delta \int_{a^{\prime}}^{b^{\prime}} p y^{\prime 2} d x>p\left(a^{\prime}\right) y\left(a^{\prime}\right) y^{\prime}\left(a^{\prime}\right)-p\left(b^{\prime}\right) y\left(b^{\prime}\right) y^{\prime}\left(b^{\prime}\right) 。
$$

Now, the right side of this inequality does not exceed

$$
\left|p\left(a^{\prime}\right) y\left(a^{\prime}\right) y^{\prime}\left(a^{\prime}\right)\right|+\left|p\left(b^{\prime}\right) y\left(b^{\prime}\right) y^{\prime}\left(b^{\prime}\right)\right|
$$

which, according to (2.13), remains finite as $a^{\prime} \rightarrow a$. Since the left side diverges to $+\infty$ as $\mathrm{a}^{\prime} \rightarrow \mathrm{a}$, the result follows.

It remains only to prove the assertion relating to $y\left(a^{\prime}\right)=y\left(b^{\prime}\right)$. Since $y(x)$ is continuous on $a \leqslant x \leqslant b$, and $y^{\prime}(x)$ exists on $a<x<b$, with $y(a)=y(b)$, it follows that there exists $\alpha, a<\alpha<b$ such that $y^{\prime}(\alpha)=0$. Moreover, assuming $y(x)>0$ on $a<x<b$, it follows from the fact that $G(x) \geqslant 0$ that we have 


$$
y^{\prime}(x) \geqslant 0 \text { for } a<x \leqslant \alpha, y^{\prime}(x) \leqslant 0 \text { for } \alpha \leq x<b \text {. }
$$

By the first of conditions (2.12), we cannot have $y(x) \equiv y(\alpha)$ for $x$ near $a$, so that $y(x)$ is strictly increasing for $a<x<a+\varepsilon$. Hence, if $a<a^{\prime}<a+\varepsilon$ we have $y(a)<y\left(a^{\prime}\right)<y(\alpha)$. It then follows from $y(\alpha)>y\left(a^{\prime}\right)>y(b)$ that there exists $b^{\prime}$ on $\alpha<x<b$ with $y\left(b^{\prime}\right)=y\left(a^{\prime}\right)$.

A similar proof shows that the corollary is valid when the second of conditions (2.12) holds.

Before leaving this section, we want to point out that hypotheses (2.2), (2.3) could be discarded provided the class of admissible functions for (2.4) satisfied the following conditions:

$$
\left\{\begin{array}{l}
u(x) \in C, a<x<b \\
u^{\prime 2}(x) \text { is integrable for every interval }\left[a^{\prime}, b^{\prime}\right] \subset(a, b) \\
\lim _{x \rightarrow a+p h u^{2}}=\lim _{x} \rightarrow b-p h u^{2}<\infty \\
\int_{a}^{b} p^{\prime 2} d x<\infty
\end{array}\right.
$$

For this class of admissible functions, (2.4) rould be a strict inequality unless $u(x) \equiv c y(x)$, where $c=0$ unless

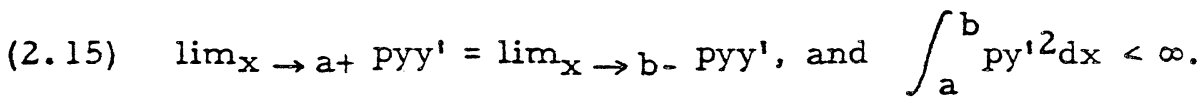

Moreover, corollary 2.1 .2 remains valid for the admissible class (2.14).

3. Extremal Properties. We consider the SturmLiouville problem

$$
\left(p y^{\prime}\right)^{\prime}+(q+\lambda g) y=0, y(a)=y(b)=0,
$$

where we assume that $p, q, g$ are continuous for $\mathrm{a}<\mathrm{x}<\mathrm{b}(-\infty \leqslant \mathrm{a}<\mathrm{b} \leqslant \infty)$, and $\mathrm{p}(\mathrm{x})>0$ on $\mathrm{a}<\mathrm{x}<\mathrm{b}$. Throughout this section we shall assume the existence of a sequence $\lambda_{1}, \lambda_{2}, \ldots$ of eigenvalues of (3.1), and a corresponding sequence $y_{1}, y_{2}, \ldots$ of eigenfunctions, each of which has only a finite number of zeros on $a<x<b$. The simple boundary conditions of (3.1) may often be replaced by less stringent conditions (which we shall note later in connection with some singular problems). 
THEOREM 3.1. Suppose that $p(x)$ satisfies conditions (2.3), that the eigenfunctions $y_{n}(x)$ of (3.1) satisfy condition (2.2) and that $y_{n}^{\prime}(x)$ is absolutely integrable on $a \leq x \leq b$, with $\int_{a}^{b}$ pys $_{n}^{2} d x<\infty$. If $\lambda_{m} \neq \lambda_{n}$, then

$$
\int_{a}^{b} g y_{m} y_{n} d x=0
$$

$\lambda=\lambda_{m}^{\text {Proof. Using the fact that } y_{m}, y_{n}}$ respectively, we obtain

$$
\left(\lambda_{n}-\lambda_{m}\right) \int_{a^{\prime}}^{b^{\prime}} g y_{n} y_{m} d x=\left.y_{n}\left(p y_{m}^{\prime}\right)\right|_{a^{\prime}} ^{b^{\prime}}-\left.y_{m}\left(p y_{n}^{\prime}\right)\right|_{a^{\prime}} ^{b^{\prime}}
$$

in the usual way. We now prove, more or less as in Theorem 2.1 , that

$$
\lim _{a^{\prime} \rightarrow a} P\left(a^{\prime}\right) y_{n}\left(a^{\prime}\right) y_{m}^{\prime}\left(a^{\prime}\right)=0,
$$

with a corresponding result as $b^{i} \rightarrow b$, whence the desired conclusion will follow from (3.2).

The proof of (3.3) makes essential use of the boundary conditions $y_{n}(a)=y_{n}(b)=0$, as well as conditions (2.2) and (2.3). On the other hand, the orthogonality conclusion of the theorem follows from (3.2) even if some or all of these conditions are not satisfied, provided

$$
\lim _{x \rightarrow a+} p\left(y_{n} y_{m}^{\prime}-y_{m} y_{n}^{\prime}\right)=\lim _{x \rightarrow b}-p\left(y_{n} y_{m}^{\prime}-y_{m} y_{h}\right)=0 .
$$

THEOREM 3.2. Let $p, q, g, y_{n}, \lambda_{n}$, satisfy the hypotheses assumed in the preceding theorem. Suppose $y_{n}$ has consecutive zeros at $x_{0}, x_{1}, \ldots, x_{k+1}$, where $a=x_{0}<x_{1}<\ldots<x_{k+1}=b$. Let $u(x)$ be any function satisfying the conditions:

( $\alpha) u^{\prime}(x)$ is absolutely integrable on $a \leq x \leq b$;

$$
(\beta) u\left(x_{i}\right)=0,0 \leqslant i \leqslant k+1 ;
$$$$
(\gamma) \int_{a}^{b} p^{\prime}{ }^{2} \mathrm{dx}<\infty, \quad \int_{\mathrm{a}}^{\mathrm{b}}|\mathrm{q}| \mathrm{u}^{2} \mathrm{~d} \mathrm{x}<\infty, 0<\int_{\mathrm{a}}^{\mathrm{b}} \mathrm{gu}{ }^{2} \mathrm{dx}<\infty .
$$

Then

$$
\lambda_{n} \leqslant\left(\int_{a}^{b} p^{\prime}{ }^{2} d x-\int_{a}^{b} q u^{2} d x\right) / \int_{a}^{b} g u^{2} d x .
$$


Moreover, equality can hold in (3.5) only if

$$
u(x) \equiv c_{i} y_{n}(x), \quad x_{i-1} \leqslant x \leqslant x_{i}, \quad 1 \leqslant i \leqslant k+1 .
$$

To prove this result, we will apply theorem 2.1 to the successive subintervals $x_{i-1}<x<x_{i}$. By our assumptions, the hypotheses of this theorem are satisfied at $x_{0}=a$ and $x_{k+1}=b$. At an interior $x_{i},(2.2)$ is also satisfied since $y_{n}^{\prime}\left(x_{i}\right)$ exists. (cf. the remark preceding the proof of theorem 2.1.) Moreover, since $\mathrm{p}(\mathrm{x})$ is continuous and positive at an interior $\mathrm{x}_{\mathrm{i}-1}$, we have

$$
0<\mathrm{k}_{\mathrm{i}-1} \leqslant \mathrm{p}(\mathrm{x}) \leqslant \mathrm{K}_{\mathrm{i}-1}
$$

in a neighbourhood of $x_{i-1}$. Hence

$$
p(x) \int_{x_{i-1}}^{x} p^{-1}(t) d t \leqslant K_{i-1} \int_{x_{i-1}}^{x} k_{i-1}^{-1} d t=O\left(x-x_{i-1}\right) .
$$

Similarly, $p(x)$ satisfies the second of conditions $(2.3)_{2}$ at $x_{i}$.

Since $u(x)$ also satisfies all the hypotheses of theorem 2.1 on the subintervals $x_{i-1} \leqslant x \leqslant x_{i}$, we have

$$
\int_{x_{i}-1}^{x_{i}}\left\{q+\lambda_{n} g\right\} u^{2} d x \leqslant \int_{x_{i-1}}^{x_{i}} p u^{\prime 2} d x, \quad 1 \leqslant i \leqslant k+1 .
$$

By Corollary 2.1.1 the equality sign holds here only if (3.6) is satisfied.

In accordance with the remarks concerning (2.14), (2.15) we may drop the boundary conditions $y_{n}(a)=y_{n}(b)=0$, as well as the conditions $(2.2)$ and $(2.3)$ for $y_{n}(x)$ and $p(x)$ respectively, provided the class of admissible functions $u(x)$ satisfy the following conditions:

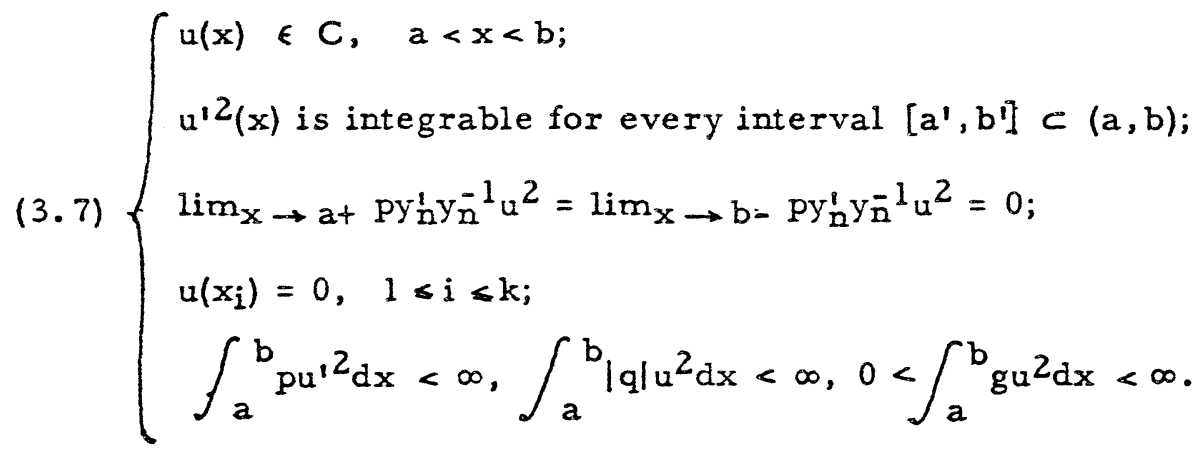


For the class of admissible functions satisfying (3.7), equality can hold in (3.5) only if $u(x)$ satisfies $(3.6)$ where $c_{1}=c_{k+1}=0$ unless

$$
\lim _{x \rightarrow a+} \operatorname{py}_{n} y_{n}^{\prime}=\lim _{x \rightarrow b-} P y_{n} y_{n}^{\prime}=0
$$

An example of a singular problem which is included in this latter formulation is the Legendre equation

$$
\left\{\left(1-x^{2}\right) y^{\prime}\right\}^{\prime}+\lambda y=0, y( \pm 1) \text { bounded, }
$$

with eigenvalues $\lambda_{n}=n(n+1), n \geqslant 0$, and corresponding eigenfunctions $P_{n}(x)$ having $n$ zeros on $-1<x<1$. In this case, the third of conditions (3.7) is satisfied if $u(x)$ is bounded near $x= \pm 1$, while condition (3.8) is also satisfied. Here our conclusion is that if $u(x)$ is any admissible function (in particular, $u(x)$ should be bounded near $x= \pm 1$, and $u(x)$ should vanish where $P_{n}(x)=0$ ), then

$$
n(n+1) \leq \int_{-1}^{1}\left(1-x^{2}\right) u^{2} d x / \int_{-1}^{1} u^{2} d x
$$

equality holding only if $u(x)$ is a (piecewise) multiple of $P_{n}(x)$ on the subintervals between successive zeros of $P_{n}(x)$.

THEOREM 3.3. Under the preceding hypotheses on $p, q, g$, and on the eigenfunctions $y_{n}(x)$ corresponding to the eigenvalue $\lambda_{n}$ of (3.1), suppose in addition, that

$\lambda_{1}<\lambda_{2}<\ldots<\lambda_{n}<\lambda_{n+1}<\ldots$, and that $y_{n}(x)$ has $k_{n} \leqslant n-1$ zeros on the open interval $a<x<b$. Finally, we assume that

$$
\int_{a}^{b} p y_{1}^{2} d x<\infty, \quad \int_{a}^{b}|q| y_{i}^{2} d x<\infty, 0<\int_{a}^{b} g y_{1}^{2} d x<\infty, 1 \leqslant i \leqslant n .
$$

Let $U(x)$ be any function satisfying the hypotheses $(\alpha),(\gamma)$ of theorem 3.2 , as well as the orthogonality conditions

$$
\int_{a}^{b} \mathrm{gy}_{i} \mathrm{Udx}=0, i=1,2, \ldots, n-1 \text {. }
$$

Then

$$
\lambda_{\mathrm{n}} \leqslant\left(\int_{\mathrm{a}}^{\mathrm{b}} \mathrm{pU^{ \prime }}{ }^{2} \mathrm{dx}-\int_{\mathrm{a}}^{\mathrm{b}} \mathrm{qU^{2 }} \mathrm{dx}\right) / \int_{\mathrm{a}}^{\mathrm{b}} \mathrm{gU^{2 }} \mathrm{dx} \text { for } \mathrm{n} \geqslant 2 .
$$

Moreover, equality holds in (3.10) if, and only if $\mathrm{U}(\mathrm{x}) \equiv \mathrm{cy} \mathrm{y}_{\mathrm{n}}(\mathrm{x}), \mathrm{a} \leqslant \mathrm{x} \leq \mathrm{b}$. 
We begin the proof by constructing a function $u(x)$ which satisfies the hypotheses of theorem 3.2. To this end, set

$$
u(x)=\sum_{i=1}^{n-1} c_{i} y_{i}(x)-U(x) \text {. }
$$

Suppose the zeros of $y_{n}(x)$ are $a=x_{0}<x_{1}<\ldots<x_{k}<x_{k+1}=b$, where $k \leqslant n-1$. Since $u(a)=u(b)=0$ for any $c_{i}, u(x)$ will satisfy $(\beta)$ if and only if the $c_{i}$ can be chosen so that

$$
\sum_{i=1}^{n-1} c_{i} y_{i}\left(x_{j}\right)=U\left(x_{j}\right), j=1,2, \ldots, k \text {. }
$$

If all $U\left(x_{j}\right)=0$, we may take all $c_{i}=0$; in this case $(3.10)$ is a consequence of theorem 3.2. Hence we may assume that (3.12) is a non-homogeneous system. We defer until later the proof that this system has a (necessarily non-trivial) solution.

With $u(x)$ - assumed defined by (3.11), (3.12) - satisfying $(\beta)$, we observe that condition $(\alpha)$ of theorem 3.2 is also satisfied by $u(x)$ in virtue of our hypotheses concerning the $y_{i}(x)$. It remains to verify $(\gamma)$; in doing so we shall note certain auxiliary results. From (3.11) we have

$$
\begin{aligned}
\int_{a}^{b} p u^{\prime 2} d x=\sum_{1}^{n-1} c_{i}^{2} \int_{a}^{b} p y_{i}^{2} d x+\int_{a}^{b} p U^{\prime 2} d x+ \\
+2 \sum_{i \neq j} c_{i} c_{j} \int_{a}^{b} p y_{i}^{\prime} y_{j}^{\prime} d x \\
-2 \sum_{1}^{n-1} c_{i} \int_{a}^{b} p U^{\prime} y_{i}^{\prime} d x .
\end{aligned}
$$

However,

$$
\begin{aligned}
\int_{a^{\prime}}^{b^{\prime}} p y_{i}^{\prime} y_{j}^{\prime} d x & =\left.p y_{i}^{\prime} y_{j}\right|_{a^{\prime}} ^{b^{\prime}}-\int_{a^{\prime}}^{b^{\prime}} y_{j}\left(p y_{1}^{\prime}\right)^{\prime} d x \\
& =\left.p y_{i}^{\prime} y_{j}\right|_{a^{\prime}} ^{b^{\prime}}+\int_{a^{\prime}}^{b^{\prime}}\left\{q+\lambda_{i} g\right\} y_{i} y_{j} d x .
\end{aligned}
$$

As $a^{\prime} \rightarrow a$ and $b^{\prime} \rightarrow b$, the right side of this equation tends to $\int_{a}^{b} q_{i} y_{j} d x$ by theorem 3.1 and equation (3.3). This latter integral exists as is easily seen by the Cauchy-Schwarz inequality. Thus we have 


$$
\int_{a}^{b} p_{i} y_{j}^{\prime} d x=\int_{a}^{b} q y_{i} y_{j} d x, \quad i \neq j
$$

Similarly, using (3.9) we obtain

$$
\int_{a}^{b} p U^{\prime} y_{i}^{\prime} d x=\int_{a}^{b} q^{b} y_{i} d x, \quad 1 \leqslant i \leqslant n-1 .
$$

(Here we used the conditions $U(a)=U(b)=0$.) We now have

$$
\begin{gathered}
\int_{a}^{b} p^{\prime 2} d x \\
(3.15)=\sum_{1}^{n-1} c_{i}^{2} \int_{a}^{b} p y_{i}^{2} d x+\int_{a}^{b} p U^{\prime 2} d x+2 \sum_{i \neq j} c_{i} c_{j} \int_{a}^{b} q y_{i} y_{j} d x \\
-2 \sum_{1}^{n-1} c_{i} \int_{a}^{b} q^{2} y_{\dot{i}} d x<\infty .
\end{gathered}
$$

A similar expansion together with several applications of the Cauchy-Schwarz inequality also gives

$$
\int_{a}^{b}|q| u^{2} d x<\infty
$$

Note that

$$
\int_{a}^{b} p^{\prime}{ }^{2} d x-\int_{a}^{b} q u^{2} d x
$$

$$
=\sum_{1}^{\mathrm{n}-1} \mathrm{c}_{1}^{2}\left\{\int_{\mathrm{a}}^{\mathrm{b}} \mathrm{py}_{\mathrm{i}}^{2} \mathrm{dx}-\int_{\mathrm{a}}^{\mathrm{b}} \mathrm{qy}_{\mathrm{i}}^{2} \mathrm{dx}\right\}+\int_{\mathrm{a}}^{\mathrm{b}} \mathrm{pU^{ \prime }} \mathrm{dx}^{2}-\int_{\mathrm{a}}^{\mathrm{b}} \mathrm{q} \mathrm{U}^{2} \mathrm{dx} .
$$

Finally, applying theorem 3.1 and (3.9), we obtain

$$
\int_{a}^{b} g u^{2} d x=\sum_{1}^{n-1} c_{i}^{2} \int_{a}^{b} g y_{i}^{2} d x+\int_{a}^{b} g U^{2} d x,
$$

and hence

$$
0<\int_{a}^{b} g u^{2} d x<\infty
$$

by our hypotheses on $U$ and the $y_{i}$.

It now follows from theorem $3.2,(3.16),(3.17)$ that

$$
\lambda_{n} \leqslant \frac{\sum_{1}^{n-1} c_{i}^{2}\left\{\int_{a}^{b} p y_{i}^{2} d x-\int_{a}^{b} q y_{i}^{2} d x\right\}+\int_{a}^{b} p U^{2} d x-\int_{a}^{b} q U^{2} d x}{\sum^{n-1} c_{i}^{2} \int_{a}^{b} g y_{i}^{2} d x+\int_{a}^{b} g U^{2} d x} .
$$


On the other hand, theorem 3.2 also gives

$$
\int_{a}^{b}{ }^{b} y_{i}^{2} d x-\int_{a}^{b} q y_{1}^{2} d x=\lambda_{i} \int_{a}^{b} g y_{i}^{2} d x, \quad 1 \leqslant i \leqslant n-1,
$$

so that

$$
\begin{aligned}
\lambda_{n} \leqslant \frac{\sum_{1}^{n-1} \lambda_{i} c_{i}^{2} \cdot \int_{a}^{b} g y_{i}^{2} d x+\int_{a}^{b} p U^{\prime 2} d x-\int_{a}^{b} q U^{2} d x}{\sum_{1}^{n-1} c_{i}^{2} \int_{a}^{b} g y_{i}^{2} d x+\int_{a}^{b} g U^{2} d x} \\
=\frac{\sum^{n-1} \lambda_{i} A_{i}+N}{\sum_{1}^{n-1} A_{i}+D},
\end{aligned}
$$

where all $A_{i} \geqslant 0, D>0$. Using the fact that $\lambda_{i}-\lambda_{n}<0$, and that at least one $A_{i}>0$ (in the case we are considering), this inequality implies that

$$
\lambda_{n}<N / D
$$

proving (3.10) with strict inequality.

We now prove that the non-homogeneous system (3.12) has a solution. The matrix of coefficients of the se equations is the $k \times(n-1)$ matrix $A$ with elements $a_{i j}$ given by

$$
a_{i j}=y_{j}\left(x_{i}\right)
$$

If $r(A)=k$, the system has a solution. Suppose $r(A)=r<k$. Then any $(r+1)$ columns of $A$ are linearly dependent, and there are constants $c_{1}, \ldots, c_{r+1}$ not all zero such that

$$
\sum_{i=1}^{r+1} c_{i} y_{i}\left(x_{j}\right)=0, \quad 1 \leqslant j \leqslant k \text {. }
$$

Define $v(x)=\sum_{i=1}^{x+1} c_{i} y_{i}(x)$. Then $v(a)=v\left(x_{1}\right)=\ldots=v\left(x_{k}\right)$

$=v(b)=0$. As in the details following equation (3.12), one sees that $v(x)$ is an admissible function for the minimum problem (3.5) so that

$$
\lambda_{n} \leqslant\left(\int_{a}^{b} p v^{\prime 2} d x-\int_{a}^{b} q v^{2} d x\right) / \int_{a}^{b} g u^{2} d x=0 .
$$


On the other hand, we now show that, in fact,

$$
\lambda_{1} \leqslant Q \leqslant \lambda_{r+1} \leqslant \lambda_{k}<\lambda_{n} ;
$$

so that $r(A)=k$ follows from this contradiction. To see this one shows, precisely as in equations (3.13) - (3.17), that

$$
\begin{aligned}
Q & =\sum_{1}^{r+1} c_{i}^{2} \lambda_{i} \int_{a}^{b} g y_{i}^{2} d x / \sum_{1}^{r+1} c_{i}^{2} \int_{a}^{b} g y_{i}^{2} d x \\
& =\sum_{1}^{r+1} d_{i} \lambda_{i},
\end{aligned}
$$

where $d_{i}=A_{i} / \sum_{1}^{r+1} A_{j}, A_{i}=c_{i}^{2} \int_{a}^{b} g y_{i}^{2} d x$, so that all $d_{i} \geqslant 0$, and $\sum_{1}^{r+1} d_{i}=1$.

By hypothesis,

whence

$$
\lambda_{1} \leqslant \lambda_{i} \leqslant \lambda_{r+1}, \quad 1 \leqslant i \leqslant r+1
$$

$$
\lambda_{1}=\lambda_{1} \sum_{1}^{r+1} d_{i} \leqslant \sum_{1}^{r+1} \lambda_{i} d_{i} \leqslant \lambda_{r+1} \sum_{1}^{r+1} d_{i}=\lambda_{r+1},
$$

establishing our contradiction.

It only remains to discuss the possibility of equality in (3.10). We have shown this can only occur when $U(x)$ also satisfies condition $(\beta)$ of theorem 3.2. By this same theorem, equality can then hold in (3.10) only if

$$
U(x) \equiv c_{i} y_{n}(x), \quad x_{i-1} \leqslant x \leqslant x_{i}, \quad 1 \leqslant i \leqslant k+1
$$

We now show that the only such $U(x)$ which also satisfy conditions (3.9) have all $c_{i}$ equal. This will complete the proof of our theorem. Suppose then that $U(x)$ satisfies both $(3.18)$ and (3.9). Then

$$
\sum_{i=1}^{k+1} c_{i} \int_{x_{i-1}}^{x_{i}} g y_{j} y_{n} d x=0, \quad 1 \leqslant j \leqslant n-1
$$

Now,

$$
\int_{a}^{b} g y_{j} y_{n} d x=\sum_{i=1}^{k+1} \int_{x_{i-1}}^{x_{i}} g y_{j} y_{n} d x=0
$$

by theorem 3.1. Multiplying the last equation by $c_{1}$ and substracting. from each of the preceding equations gives 


$$
\sum_{i=2}^{k+1}\left(c_{i}-c_{1}\right) \int_{x_{i-1}}^{x_{i}} g y_{j} y_{n} d x=0,1 \leq j \leqslant n-1
$$

Since $k \leqslant n-1$, this system of $n-1$ equations has only the trivial solution $c_{i}-c_{1}=0$, unless the determinant of coefficients of the first $k$ equations is zero. However, if this determinant were zero there would exist constants $a_{1}, \ldots, a_{k}$, not all zero, such that

$$
\sum_{j=1}^{k} a_{j} \int_{x_{i-1}}^{x_{i}} g y_{j} y_{n} d x=0, \quad 2 \leqslant i \leqslant k+1
$$

Setting $u(x)=\sum_{j=1}^{k} a_{j} y_{j}(x)$, this implies

$$
\int_{x_{i-1}}^{x_{i}} \operatorname{gy} u d x=0, \quad 2 \leqslant i \leqslant k+1
$$

Hence we have $\int_{x_{1}}^{b} g_{n} u d x=0$, and since $\int_{a}^{b} g_{n} u d x=0$ by

Theorem 3.1, we also have

$$
\int_{a}^{x_{1}} g y_{n} u d x=0
$$

According to $(3.20)$ and $(3.21)$ we now have

$$
\int_{a}^{x_{i}} \operatorname{gy}_{n} u d x=0, \quad 1 \leqslant i \leqslant k+1 \text {. }
$$

Now, as in theorem 3.1, we have

$$
-\left.p y_{j} y_{n}^{\prime}\right|_{a} ^{x_{i}}=\left(\lambda_{n}-\lambda_{j}\right) \int_{a}^{x_{i}} g y_{n} y_{j} d x, 1 \leqslant j \leqslant k, \quad 1 \leqslant i \leqslant k+1 .
$$

Using (3.3) this reduces to

$$
-p\left(x_{i}\right) y_{n}^{\prime}\left(x_{i}\right) y_{j}\left(x_{i}\right) /\left(\lambda_{n}-\lambda_{j}\right)=\int_{a}^{x_{i}} g y_{n} y_{j} d x .
$$

Multiply this equation by $a_{j}$ and sum over $1 \leqslant j \leqslant k$ to obtain $-p\left(x_{j}\right) y_{n}^{\prime}\left(x_{i}\right) \sum_{j=1}^{k} a_{j} y_{j}\left(x_{j}\right) /\left(\lambda_{n}-\lambda_{j}\right)=\int_{a}^{x_{i}} g y_{n} u d x=0,1 \leqslant i \leqslant k+1$,

by (3.22). However, for $1 \leqslant i \leqslant k$, we have $p\left(x_{i}\right)>0, y_{n}^{\prime}\left(x_{j}\right) \neq 0$, hence 
By hypothesis, not all $a_{j} /\left(\lambda_{n}-\lambda_{j}\right)$ are zero, and hence the determinant $\left|y_{j}\left(x_{j}\right)\right|=0$. But this is impossible since we have already established that $r\left(\left\|y_{j}\left(x_{i}\right)\right\|\right)=k$.

As before, the boundary conditions $y_{j}(a)=y_{j}(b)=0$ may be omitted, as well as condition (2.2) for $y_{j}(x), 1 \leqslant j \leqslant n$, and conditions (2.3) for $p(x)$, provided the $y_{j}(x)$ satisfy the remaining hypotheses of Theorem 3.3, together with the conditions

(3.24) $\lim _{x \rightarrow a+} P y_{n}^{1} y_{n}^{-1} y_{j}^{2}=\lim _{x \rightarrow b-} y_{n}^{1} y_{n}^{-1} y_{j}^{2}=0,1 \leqslant j \leqslant n-1$,

(3.25) $\lim _{x \rightarrow a+}$ pyll $_{j}=\lim _{x \rightarrow b-p y l} y_{j}=0,1 \leqslant i, j \leqslant n$.

In this case, the minimum property $(3.10)$ holds for the class of functions $U(x)$ satisfying the conditions (cf. (3.7)):

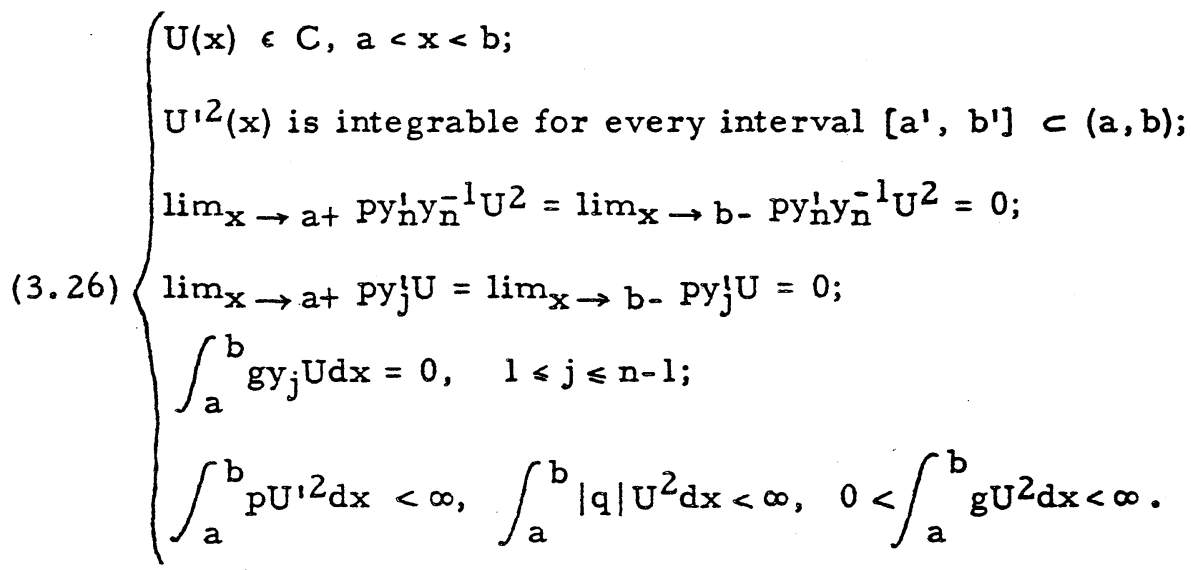

For this class of admissible functions, equality holds in (3.10) if, and only if, $\mathrm{U}(\mathrm{x}) \equiv \mathrm{cy}_{\mathrm{n}}(\mathrm{x})$.

The Legendre eigenvalue problem, as stated previously, satisfies hypotheses $(3.24),(3.25)$, and again the limit conditions of $(3.26)$ are satisfied if $U(x)$ is bounded near $x= \pm 1$. Hence we conclude that if $U(x)$ is any (suitably integrable) function, continuous and bounded on $-1<x<1$, such that

$$
\int_{-1}^{1} P_{j}(x) U(x) d x=0, \quad 0 \leqslant j \leqslant n-1,
$$


then

$$
n(n+1) \leqslant \int_{-1}^{1}\left(1-x^{2}\right) U^{\prime} 2 d x / \int_{-1}^{1} U^{2} d x
$$

equality holding only if $U(x) \equiv c P_{n}(x)$.

As a second singular problem, this time on an infinite interval, we consider the Hermite equation

$$
\left(e^{-x^{2}} y^{\prime}\right)^{\prime}+\lambda e^{-x^{2}} y=0, \quad-\infty<x<\infty,
$$

with boundary condition $y=O\left(|x|^{k}\right)$ as $|x| \rightarrow \infty$, for some $k>0$. Here the eigenvalues are $\lambda_{n}=2(n-1), n=1,2, \ldots$, with corresponding eigenfunctions the Hermite polynomials $y_{n}=H_{n-1}(x)$ having $n-1$ zeros on $-\infty<x<\infty$. Conditions (3.24), (3.25) are clearly satisfied, as are the pertinent conditions of (3.26) for functions $U(x)$ satisfying the boundary conditions noted above.

For a final example, consider the equation

$$
y^{\prime \prime}+\left\{\lambda-\left(m^{2}-\frac{1}{4}\right) x^{-2}\right\} y=0, y(0)=y(1)=0, \quad\left(m \geq \frac{1}{2}\right)
$$

The eigenvalues are given [4, p. 325] by $\lambda_{n}=k_{n}^{2}, n=1,2, \ldots$, where $k_{n}$ is the $n^{t h}$ positive zero of the Bessel function $J_{m}(x)$. The corresponding eigenfunction is $y_{n}=x^{\frac{1}{2}} J_{m}\left(k_{n} x\right)$ with $n-1$ zeros on $0<x<1$. In this case the boundary conditions are satisfied by all $y_{n}$, as indeed are all the hypotheses of theorem 3.3. We conclude that

$$
\mathrm{k}_{\mathrm{n}}^{2} \leqslant\left\{\int_{0}^{1} \mathrm{U}^{2} \mathrm{dx}+\int_{0}^{1}\left(\mathrm{~m}^{2}-\frac{3}{4}\right) \mathrm{x}^{-2} \mathrm{U}^{2} \mathrm{dx}\right\} / \int_{0}^{1} \mathrm{U}^{2} \mathrm{dx}
$$

whenever $\int_{0}^{1} x^{\frac{1}{2}} J_{m}\left(k_{i} x\right) U(x) d x=0$ for $i=1,2, \ldots, n-1$, equality holding only if $U=x^{\frac{1}{2}} J_{m}\left(k_{n} x\right)$.

4. Maximum-minimum properties of the eigenvalues. Theorem 3.2 requires a precise knowledge of the zeros of the $n^{\text {th }}$ eigenfunction $y_{n}(x)$. If, however, we assume that the $\lambda_{i}$ and $y_{i}$ satisfy the additional hypotheses of theorem 3.3 , we may avoid this requirement as we now show. Let

$$
\begin{gathered}
K_{n}=\{u(x) \mid u(x) \text { has no more than }(n-1) \text { zeros on } a<x<b ; \\
u(a)=u(b)=0\},
\end{gathered}
$$




$$
\begin{gathered}
K_{n}(u)=\{v(x) \mid v \text { satisfies }(\alpha),(\gamma) \text { of theorem 3.2; } \\
v=0 \text { if } u=0\} .
\end{gathered}
$$

Set

(4.1) $d_{n}(u)=\inf _{v} \in K_{n}(u)\left\{\left(\int_{a}^{b}{ }_{p v^{\prime}} 2 d x-\int_{a}^{b} q^{2} d x\right) / \int_{a}^{b} g v^{2} d x\right\}$. Then ${ }^{1)}$

$$
\lambda_{\mathrm{n}}=\sup _{\mathrm{u} \in \mathcal{K}_{\mathrm{n}}} \mathrm{d}_{\mathrm{n}}(\mathrm{u}) .
$$

To prove this result, we note that since $y_{n}$ is assumed to have $k \leqslant n-1$ zeros on $a<x<b$, we have

$$
\lambda_{n}=d_{n}\left(y_{n}\right) \leqslant \sup _{u} \in \kappa_{n} d_{n}(u),
$$

by theorem 3.2. To prove the opposite inequality, let $u \in K_{n}$, and suppose the zeros of $u$ are $a, x_{1}^{1}, x_{2}^{1}, \ldots, x_{\alpha}^{1}, b$, where $\alpha \leq n-1$. We now construct a function $v \in \mathcal{K}_{n}(u)$ whose Rayleigh quotient appearing in (4.1) does not exceed $\lambda_{n}$. This will prove $d_{n}(u) \leq \lambda_{n}$ for all $u \in K_{n}$, whence the opposite inequality to (4.3) follows. In fact, it suffices to take

$$
v(x)=\sum_{1}^{n} c_{i} y_{i}(x) \text {. }
$$

As in the proof of theorem 3.3,v $\in \mathbb{K}_{n}(u)$ if the $c_{i}$ (not all zero) can be chosen so that

$$
\sum_{i=1}^{n} c_{i} y_{i}\left(x_{j}^{j}\right)=0, \quad 1 \leq j \leqslant \alpha(\leqslant n-1) .
$$

Such a solution always exists for this homogeneous system. But then, precisely as in the case of the function $v(x)$ of theorem 3.3, it follows that

$$
\lambda_{1} \leqslant\left(\int_{a}^{b} \mathrm{pv}^{\prime} 2 \mathrm{dx}-\int_{\mathrm{a}}^{\mathrm{b}} \mathrm{qv} \mathrm{v}^{2} \mathrm{dx}\right) / \int_{\mathrm{a}}^{\mathrm{b}} \mathrm{gv} \mathrm{d}^{2} \mathrm{dx} \leqslant \lambda_{\mathrm{n}},
$$

completing the proof of (4.2).

1) This result is attributed by $R$. Courant [4, p. 463, footnote] to $\mathrm{K}$. Hohenemser. 
A second maximum-minimum characterization of $\lambda_{n}$ is due to $R$. Courant $[4$, p. 406] . We formulate it as follows: Let $\mathcal{L}_{\mathrm{n}}$ be the class of all $(\mathrm{n}-1)$-tuples $\left\{v_{1}(\mathrm{x}), \ldots, v_{\mathrm{n}-1}(\mathrm{x})\right\}$ of functions such that $\int_{a}^{b} g v_{i}^{2} \mathrm{dx}<\infty, 1 \leqslant i \leqslant n-1$. Let $\mathcal{L}_{n}\left(v_{1}, \ldots, v_{n-1}\right)=\{u(x) \mid$ u satisfies $(\alpha),(\gamma)$ of theorem 3.2;

$$
\begin{aligned}
& u(a)=u(b)=0 ; \\
& \left.\int_{a}^{b} \operatorname{guv}_{\dot{i}} d x=0, \quad l \leq i \leq n-1\right\},
\end{aligned}
$$

and set

(4.4) $d_{n}\left(v_{1}, \ldots, v_{n-1}\right)$

$$
=\inf _{\mathrm{u}} \in \mathcal{L}_{\mathrm{n}}\left(\mathrm{v}_{1}, \ldots, \mathrm{v}_{\mathrm{n}-1}\right)\left\{\left(\int_{\mathrm{a}}^{\mathrm{b}} \mathrm{pu}^{\prime 2} \mathrm{dx}-\int_{\mathrm{a}}^{\mathrm{b}} \mathrm{qu} \mathrm{z}^{2} \mathrm{dx}\right) / \int_{\mathrm{a}}^{\mathrm{b}} \mathrm{gu} \mathrm{L}_{\mathrm{dx}}\right\} .
$$

Then

(4.5) $\left.\quad \lambda_{n}=\sup _{\left(v_{1}\right.}, \ldots, v_{n-1}\right) \in \mathcal{L}_{n} d_{n}\left(v_{1}, \ldots, v_{n-1}\right)$. so that

For, by theorem 3.3 we have $d_{n}\left(y_{1}, \ldots, y_{n-1}\right)=\lambda_{n}$,

$$
\left.\lambda_{n} \leqslant \sup _{\left(v_{1}\right.}, \ldots, v_{n-1}\right) \in \mathcal{L}_{n} d_{n}\left(v_{1}, \ldots, v_{n-1}\right) .
$$

Now for any $\left(v_{1}, \ldots, v_{n-1}\right) \in \mathcal{L}_{n}$, define the function

$$
\mathrm{u}(\mathrm{x})=\sum_{1}^{\mathrm{n}} \mathrm{c}_{\mathbf{i}} \mathrm{y}_{\mathbf{i}}(\mathrm{x}) \text {, }
$$

where the $c_{i}$ are any non-trivial solution of the n-1 homogeneous equations

$$
\int_{a}^{b} \operatorname{guv}_{j} \mathrm{dx}=\sum_{i=1}^{n} c_{i} \int_{a}^{b} g_{j} y_{i} d x=0,1 \leqslant j \leqslant n-1 .
$$

Then $u \in \mathcal{L}_{n}\left(v_{1}, \ldots, v_{n-1}\right)$, and as before $d_{n}\left(v_{1}, \ldots, v_{n-1}\right) \leqslant \lambda_{n}$, completing the proof of $(4.5)$. 


\section{REFERENCES}

1. M. Bocher, Leçons sur les méthodes de Sturm, (Paris, 1917).

2. E. Coddington and N. Levinson, Theory of Ordinary Differential Equations, (New York, 1955).

3. L. Collatz, Eigenwertprobleme und ihre numerische Behandlung, (New York, 1948).

4. R. Courant, and D. Hilbert, Methods of Mathematical Physics, vol. 1, (New York, 1953).

McMaster University 